\title{
Are we making good use of our public resources? The false-positive rate of screening by fundus photography for diabetic macular oedema
}

\author{
Raymond LM Wong *, CW Tsang, David SH Wong, Sarah McGhee, CH Lam, J Lian, Jacky WY Lee, \\ Jimmy SM Lai, Victor Chong, lan YH Wong *
}

\section{A B S T R A C T}

Introduction: A large proportion of patients diagnosed with diabetic maculopathy using fundus photography and hence referred to specialist clinics following the current screening guidelines adopted in Hong Kong and United Kingdom are found to be false-positive, implying that they did not have macular oedema. This study aimed to evaluate the false-positive rate of diabetic maculopathy screening using the objective optical coherence tomography scan.

Methods: This was a cross-sectional observational study. Consecutive diabetic patients from the Hong Kong West Cluster Diabetic Retinopathy Screening Programme with fundus photographs graded R1M1 were recruited between October 2011 and June 2013. Spectral-domain optical coherence tomography imaging was performed. Central macular thickness of $\geq 300 \mu \mathrm{m}$ and/or the presence of optical coherence tomography signs of diabetic macular oedema were used to define the presence of diabetic macular oedema. Patients with conditions other than diabetes that might affect macular thickness were excluded. The mean central macular thickness in various subgroups of R1M1 patients was calculated and the proportion of subjects with central macular thickness of $\geq 300 \mu \mathrm{m}$ was used to assess the falsepositive rate of this screening strategy.

Results: A total of 491 patients were recruited during the study period. Of the 352 who were eligible for analysis, $44.0 \%, 17.0 \%$, and $38.9 \%$ were graded as M1 due to the presence of foveal 'haemorrhages', 'exudates', or 'haemorrhages and exudates', respectively. The mean ( \pm standard deviation) central macular thickness was $265.1 \pm 55.4 \mu \mathrm{m}$. Only $13.4 \%$ (95\% confidence interval, $9.8 \%-17.0 \%$ ) of eyes had a central macular thickness of $\geq 300 \mu \mathrm{m}$, and $42.9 \%$

This article was published on $7 \mathrm{Jul}$ 2017 at www.hkmj.org. (95\% confidence interval, $37.7 \%-48.1 \%$ ) of eyes had at least one optical coherence tomography sign of diabetic macular oedema. For patients with retinal haemorrhages only, 9.0\% (95\% confidence interval, $4.5 \%-13.5 \%)$ had a central macular thickness of $\geq 300 \mu \mathrm{m} ; 23.2 \%$ (95\% confidence interval, 16.6\%29.9\%) had at least one optical coherence tomography sign of diabetic macular oedema. The false-positive rate of the current screening strategy for diabetic macular oedema was $86.6 \%$.

Conclusion: The high false-positive rate of the current diabetic macular oedema screening adopted by the United Kingdom and Hong Kong may lead to unnecessary psychological stress for patients and place a financial burden on the health care system. A better way of screening is urgently needed. Performing additional spectral-domain optical coherence tomography scans on selected patients fulfils this need.

\section{Hong Kong Med J 2017;23:356-64}

DOI: $10.12809 / \mathrm{hkmj} 166078$

1,2,3 RLM Wong *, MRCSEd (Ophth), FCOphth HK

${ }_{1,3}$ CW Tsang, FRCSEd (Ophth)

2 DSH Wong, FRCOphth

${ }^{4} \mathrm{~S}$ McGhee, FFPH (UK)

${ }^{2} \mathrm{CH}$ Lam, BSc(Hons) in Optometry

${ }^{4} \mathrm{~J}$ Lian, PhD

2 JWY Lee, FRCSEd (Ophth)

2 JSM Lai, FRCOphth

${ }^{2,5}$ V Chong, FRCOphth

${ }^{2}$ IYH Wong *, FRCOphth

1 Department of Ophthalmology and Visual Sciences, The Chinese University of Hong Kong, Shatin, Hong Kong

Department of Ophthalmology, The University of Hong Kong, Pokfulam, Hong Kong

Hong Kong Eye Hospital, 147K Argyle Street, Hong Kong

${ }^{4}$ Department of Community Medicine, The University of Hong Kong,

Pokfulam, Hong Kong

5 Oxford Eye Hospital, Oxford University Hospitals, Oxford, United Kingdom

* Corresponding authors: raymondwlm@hotmail.com, wongyhi@hku.hk

New knowledge added by this stud

- The current Hong Kong diabetic retinopathy screening results in a high level of false positive results, which in turn creates unnecessary psychological stress for patients and financial burden on our health care system.

- The current screening programme can be improved by the use of optical coherence tomography scans in selected patients.

Implications for clinical practice or policy

- The results of our study reflect a need to revise the current Hong Kong diabetic retinopathy screening system (Risk Assessment and Management Programme; RAMP-DR). 


\section{Introduction}

Diabetic retinopathy (DR) is one of the most common causes of blindness and its incidence increases with the duration of diabetes. ${ }^{1-3}$ The reported prevalence ranges from $24 \%-40 \%$ after 5 years to $80 \%-90 \%$ after 20 years of diabetes. ${ }^{2-4}$ Diabetic macular oedema (DME) and proliferative diabetic retinopathy (PDR) are the two major causes of vision loss in DR. ${ }^{5}$ The Early Treatment Diabetic Retinopathy Study (ETDRS) showed that clinically significant macular oedema (CSME) leads to moderate vision loss in one of four patients with this condition over 3 years. Timely laser treatment reduces the risk of vision loss by half. ${ }^{6}$ In recent years, there has been a move towards the use of newer treatment modalities, such as intravitreal injection of anti-vascular endothelial growth factor (VEGF) agents that are superior to the traditional laser treatment in the management of CSME. ${ }^{7-14}$ Screening for DR has been proven to be cost-effective in reducing significant vision loss by early detection of the pathology. ${ }^{15-17}$ This will subsequently reduce the financial burden caused by vision complications of DR on the health care system. ${ }^{18,19}$ A number of DR screening strategies are available with different efficacies. ${ }^{20}$ Systematic screening for DR with fundus photography has been implemented in the UK and Hong Kong, and it has been shown to be cost-effective for sight-threatening conditions from the provider's perspective (Fig 1). ${ }^{21}$ However, the accuracy of the current DR screening protocol for DME remains unknown. With limited health care resources, improving the accuracy and cost-effectiveness of systematic screening programmes is important. $^{22}$

In Hong Kong, individuals who attend public out-patient clinics for diabetes management are

\section{我們是否善用公共資源？眼底攝影術診斷糖尿病 黃斑水腫的假陽性率}

黃禮文、曾智偉、黃世雄、麥潔儀、林展行、連金曉、 李偉業、黎少明、莊毅恆、王逸軒

引言：根據目前香港和英國的指引使用眼底攝影術作為糖尿病黃斑水 腫的篩查方法, 發現大部分因此而轉介到專科門診的病人均有假陽性 結果。本研究旨在使用光學相幹斷層掃描技術（OCT）來評估糖尿病 黃斑病變篩查的假陽性率。

方法：這觀察橫斷面研究的對象是2011年10月至2013年6月參與港 島西聯網糖尿病視網膜病變檢查計劃, 眼底攝影結果被評為視網膜疾 病 $\mathrm{R} 1$ 期和黃斑病變 $\mathrm{M} 1$ 期的糖尿病患者。我們為病人進行頻域OCT, 並把糖尿病黃斑水腫定義為黃斑中心視網膜厚度為 $\geq 300$ 微米和/或在 OCT 影像上出現糖尿病黃斑水腫的症狀。病人有糖尿病以外的原因而 有可能影響黃斑中心厚度均不會被列入研究範圍。我們計算 $\mathrm{R} 1 \mathrm{M} 1$ 各 組患者黃斑中心視網膜的平均厚度, 並使用厚度 $\geq 300$ 微米的參與者比 例來評估篩查的假陽性率。

結果：研究期間共招募了 491名患者。被列入研究範圍的352例中診 斷為黃斑病變 M 1 期的原因可分為三種, 它們分別為中央凹陷區出血 $(44.0 \%)$ 、滲出 $(17.0 \%)$ 以及出血和滲出 $(38.9 \%)$ 。參與者黃 斑中心視網膜的平均厚度為 265.1 微米（標準差55.4微米）。病例中 只有 $13.4 \%$ （95\%置信區間，9.8\%-17.0\%）的黃斑中心視網膜厚度 $\geq 300$ 微米, $42.9 \%$ (95\%置信區間， $37.7 \%-48.1 \%$ ) 的OCT影像至 少出現一個糖尿病黃斑水腫的症狀。對於只有視網膜出血的患者, 9.0\%（95\%置信區間，4.5\%-13.5\%）的黃斑中心視網膜厚度 $\geq 300$ 微 米, $23.2 \%$ （95\%置信區間， $16.6 \%-29.9 \%$ ) 的OCT影像上至少出 現一個糖尿病黃斑水腫的症狀。糖尿病黃斑水腫篩查的假陽性率為 $86.6 \%$ 。

結論：目前英國和香港採用的糖尿病黃斑水腫篩查方法, 其高假陽性 率可能導致患者承受不必要的心理壓力, 並對醫療體系造成經濟負 擔。尋找一個更好的穊選方法具迫切性。為指定患者進行額外的頻域 OCT可以是其中一個方法。

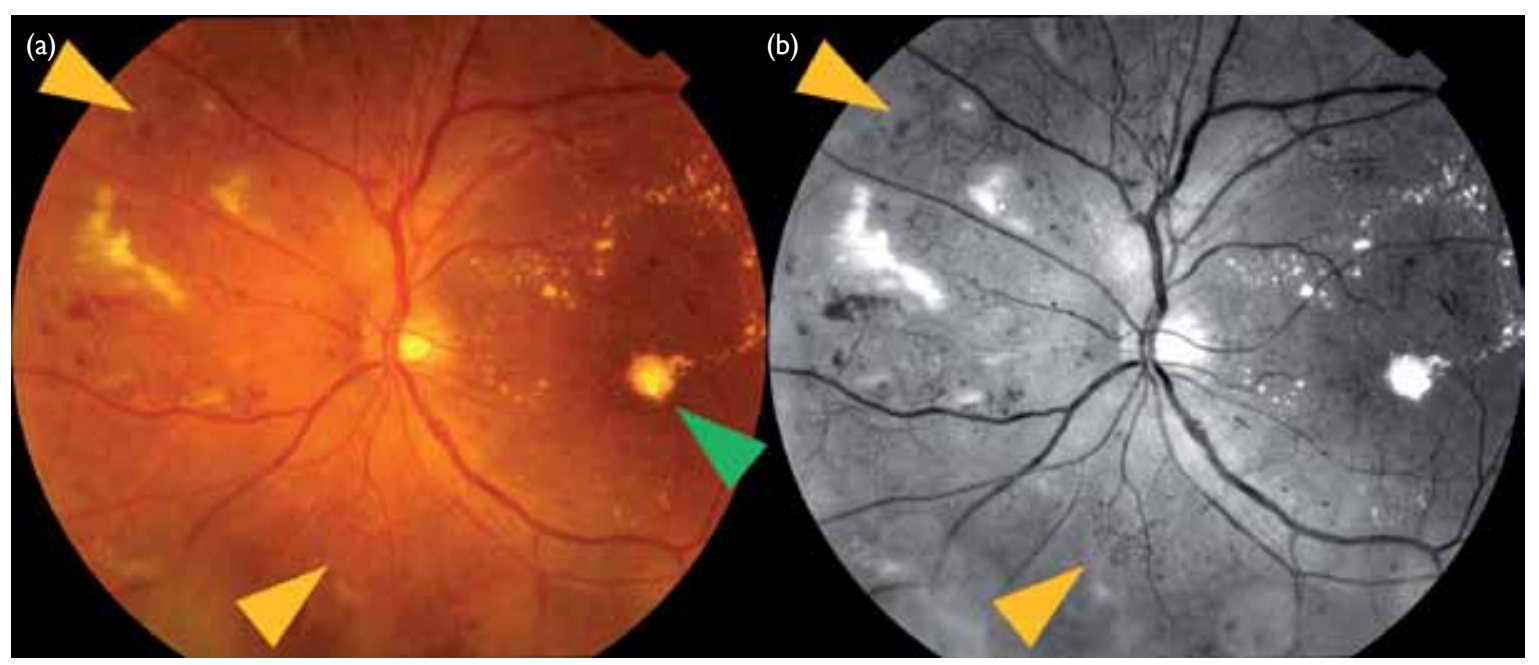

FIG I. (a) The left disc and part of the macula showing evidence of new vessels elsewhere (yellow arrowheads) and exudates (green arrowhead). (b) Presence of new vessels are better seen on a red-free version of the same photo (yellow arrowheads) 
offered annual fundus photography for DR screening. Eyes are graded according to the protocol adopted by the UK National Health Service (Diabetic Eye Screening Revised Grading Definitions, version 1.4, NHS Screening Programmes). Those found to have sight-threatening diabetic retinopathy (STDR), that is, patients who have their worse eye graded as preproliferative DR (R2 or above), maculopathy (M1) or ungradable at screening, are referred for clinical assessment by an ophthalmologist. Those confirmed to have CSME or PDR are then offered appropriate treatment. $^{6}$

Unlike PDR, DME cannot be visualised with fundus photography because of the lack of stereopsis in two-dimensional photographs. Instead of appreciating the actual macular thickening, determining the presence of surrogate markers in the macula, such as retinal exudates and haemorrhages, is currently the recommended first step in predicting the presence of macular oedema from fundus photography. ${ }^{23}$

Our unpublished data from the Hong Kong West Cluster DR Screening Programme showed that the prevalence of ungradable fundus photographs was $3.8 \%$ and the rate for a positive screen for M1 by fundus photography was $14 \%$. Those graded as M1 accounted for $86.4 \%$ of all the referred STDR cases. A similar result was found in the UK where $79 \%$ of all subjects with diabetes who were referred to ophthalmology clinics following screening were graded as $\mathrm{M} 1 .{ }^{24}$ These findings indicate that $\mathrm{M} 1$ is the most prevalent type of STDR diagnosed at screening among subjects with diabetes in both the UK and Hong Kong. Due to the limited ability of fundus photography to visualise retinal thickening in DME, the number of false positives (ie those without DME) has become a concern. The opportunity to detect M1 at an early stage during DR screening is potentially very valuable. A high false-positive rate is perceived to increase the burden on patients and public health care resources. Because these false positive cases do not need treatment, such extra workload produces no benefit and could be considered a waste of public resources. On the other hand, it would benefit the cost-effectiveness of macular oedema detection if a screening protocol with fewer false positive results could be identified.

In recent years, optical coherence tomography (OCT) has been developed to generate highly accurate and objective information regarding the cross-sectional view of the retina. This scanning technique is fast, safe, non-invasive, contact-free, and with no radiation exposure. It is a reliable means to identify macular thickening in diabetics. Comparison of photographic-graded M1 with the findings from OCT scans can perhaps enable us to better understand the current level of false positives at screening and provide essential information to evaluate the means by which the cost-effectiveness of screening for M1 can be improved. The aims of this study were to evaluate the false-positive rate of grade M1 using the existing criteria and OCT imaging as the reference standard, and also to estimate the consequences of inappropriate specialty clinic referrals generated from the false positive results.

\section{Methods}

In this cross-sectional observational study, patients were recruited from the Hong Kong West Cluster DR Screening Programme. This programme offers annual DR screening to all diabetic patients in Queen Mary Hospital (a teaching hospital in Hong Kong) and patients referred from the Hong Kong Risk Assessment and Management Programme (RAMP-DR screening) in the Hong Kong West Cluster. In other words, this programme cares for the eye conditions of all the diabetic patients attending public sector in the Hong Kong West Cluster. There are 500000 residents in the Hong Kong West Cluster and around 7000000 citizens in Hong Kong. Assuming the prevalence of diabetes mellitus to be similar across different regions of Hong Kong, Hong Kong West Cluster cares for $7.1 \%(500000 / 7000000)$ of diabetic patients in the city. All patients who attended this programme had mydriatic fundus photographs taken for DR screening. Fundus photographs were graded by a qualified RAMP screening programme grader (an optometrist) according to the UK NHS Diabetic Eye Screening-Feature Based Grading Forms (Version 1.4). This allocated an M1 grade to subjects with the presence of exudates or retinal haemorrhages/ microaneurysms within 1 disc diameter $(1.5 \mathrm{~mm})$ of the centre of the fovea, accompanied by a reduction in the best-corrected visual acuity to 6/12 or worse. In addition to maculopathy (M0-M1), retinopathy (R0-R3) was graded from the fundus photographs using the same screening standard. Nonetheless, because patients with moderate non-proliferative DR or worse (DR screening grade R2 or above), which constituted $3.0 \%$ of the screened population in Hong Kong, ${ }^{25}$ needed to be assessed and followed by ophthalmologists regardless of their maculopathy status (M0 or M1), these subjects do not contribute to the extra workload of specialist clinics. Therefore, the current study focused on only patients in whom maculopathy or mild retinopathy (R1M1) was revealed following screening with fundus photography.

Consecutive subjects aged 18 years or above (no upper age limit) with fundus photographs graded R1M1 were recruited from October 2011 to June 2013. Patients with retinal or choroidal conditions other than diabetes that could affect retinal thickness were excluded. Patients with media opacities such as cataract were not excluded 
provided the grading of fundus photography was not affected and optimal OCT scans could be obtained. Therefore, all ungradable photos were excluded from this study. Informed consent was obtained from all the patients. This study adhered to the tenets of the Declaration of Helsinki and was approved by the Institutional Review Board of the University of Hong Kong/Hong Kong West Cluster.

Because the traditional gold standard for diagnosing CSME, slit lamp biomicroscopy, is subjective and difficult to validate, we used OCT imaging as the reference standard for diagnosis. Spectral-domain OCT (sd-OCT) imaging was performed with a Carl Zeiss Cirrus sd-OCT (Carl Zeiss Meditec, Dublin [CA], United States) on all included subjects to determine central macular thickness (CMT) using the Macular Cube protocol (average retinal thickness in the area enclosed in a 1000- $\mu \mathrm{m}$ diameter circle centred at the fovea). A CMT of $300 \mu \mathrm{m}$ was used as the cut-off for normal macular thickness (the rationale of choosing this value will be discussed in detail in Discussion).

The OCT scans were analysed by an experienced retina specialist for the presence of OCT signs of macular oedema, namely the presence of intraretinal cyst, subretinal fluid, diffuse retinal thickening, or change in internal limiting membrane (ILM) contour. During analysis, the retina specialist was blinded to CMT value.

\section{Statistical analyses}

Only one eye from each subject was used in the analysis. For patients with both eyes graded as R1M1, only their right eye was chosen for analysis. A descriptive analysis was used to summarise the demographic characteristics of study subjects. The positive predictive values (PPVs) of different combinations of criteria were calculated with $95 \%$ confidence interval (CI). We first classified the fundus photographs into three groups according to the criteria used to grade them as M1 at screening: haemorrhages only, exudates only, or both haemorrhages and exudates. Each of these three groups was compared with the reference standard results of the OCT scan that measured a CMT of $\geq 300 \mu \mathrm{m}$ to calculate the PPV of each M1 criterion at screening. We also calculated the PPV by comparing each of these three groups with the reference standard results of the OCT that measured any OCT signs of DME. Chi squared test was used to determine whether there were any significant differences in the PPVs among the three groups. The false-positive rate was obtained by subtracting the PPV from 1.

\section{Results}

A total of 491 R1M1 patients were recruited during the study period. After excluding those with conditions that might affect macular thickness or the quality of an OCT scan such as dense cataract, 352 R1M1 patients remained eligible for analysis. The mean ( \pm standard deviation) age of these 352 patients was $65 \pm 11$ years and 187 (53\%) patients were female.

Among the 352 eyes analysed, 155 (44.0\%), 60 (17.0\%), and 137 (38.9\%) were graded as M1 based on the presence of foveal haemorrhages, exudates, or haemorrhages and exudates, respectively, in the fundus photographs (Table 1).

The overall mean CMT of all the subjects was $265.1 \mu \mathrm{m}$. The mean CMT was $256.8 \mu \mathrm{m}$ for the patients with haemorrhages only, $270.0 \mu \mathrm{m}$ for the patients with exudates only, and $272.4 \mu \mathrm{m}$ for those with both haemorrhages and exudates.

Overall, only $47(13.4 \%)$ of the 352 (95\% CI, 9.8\%-17.0\%) eyes had a CMT of $\geq 300 \mu \mathrm{m}$ (Table $1)$. Using the criterion of the presence of retinal haemorrhages within 1 disc diameter from the centre of the fovea, $9.0 \%$ (95\% CI, 4.5\%-13.5\%) of eyes had a CMT of $\geq 300 \mu \mathrm{m}$, which was the lowest proportion. Applying the criterion of presence of exudates at the fovea, $15.0 \%$ (95\% CI, 6.0\%-24.0\%) had a CMT of $\geq 300 \mu \mathrm{m}$; and in the presence of simultaneous haemorrhages and exudates, this figure was $17.5 \%$

TABLE I. Incidence of OCT signs among fundus photographic signs of diabetic macular oedema and corresponding CMT

\begin{tabular}{|c|c|c|c|c|c|c|c|c|}
\hline \multirow{2}{*}{$\begin{array}{l}\text { Criteria of grading as } \\
\text { M1 cases }\end{array}$} & \multicolumn{5}{|c|}{ No. $(\%)^{*}$} & \multicolumn{3}{|c|}{ No. (\%) $[95 \% \mathrm{Cl}]$ or mean \pm SD } \\
\hline & Eyes & Cyst & $\begin{array}{l}\text { Subretinal } \\
\text { fluid }\end{array}$ & $\begin{array}{c}\text { Diffuse } \\
\text { retinal } \\
\text { thickening }\end{array}$ & $\begin{array}{c}\text { ILM } \\
\text { profile } \\
\text { change }\end{array}$ & Any OCT signs & $\mathrm{CMT}(\mu \mathrm{m})$ & $\mathrm{CMT} \geq 300 \mu \mathrm{m}$ \\
\hline Haemorrhage only & $155(44.0)$ & $27(17.4)$ & $1(0.6)$ & $14(9.0)$ & $25(16.1)$ & 36 (23.2) [16.6-29.9] & $256.8 \pm 58.8$ & $14(9.0)[4.5-13.5]$ \\
\hline Exudate only & $60(17.0)$ & $22(36.7)$ & $2(3.3)$ & $17(28.3)$ & $19(31.7)$ & $31(51.7)$ [39.1-64.3] & $270.0 \pm 55.4$ & 9 (15.0) [6.0-24.0] \\
\hline Haemorrhage and exudate & $137(38.9)$ & $69(50.4)$ & $1(0.7)$ & $44(32.1)$ & $45(32.8)$ & $84(61.3)$ [53.1-69.5] & $272.4 \pm 50.4$ & $24(17.5)[11.1-23.9]$ \\
\hline All & $352(100.0)$ & $118(33.5)$ & $4(1.1)$ & $75(21.3)$ & $89(25.3)$ & $151(42.9)[37.7-48.1]$ & $265.1 \pm 55.4$ & 47 (13.4) [9.8-17.0] \\
\hline
\end{tabular}

Abbreviations: $\mathrm{Cl}=$ confidence interval; CMT = central macular thickness; ILM = internal limiting membrane; $\mathrm{MI}=$ maculopathy; $O C T=$ optical coherence tomography; SD = standard deviation

* Because of rounding, not all percentages total 100 
(95\% CI, 11.1\%-23.9\%) [Chi squared=4.70, $\mathrm{P}=0.096$ ]

When CMT was not taken into account, 151 (42.9\%) of the 352 (95\% CI, 37.7\%-48.1\%) eyes had at least one OCT sign of DME (Table 1). The proportion of eyes with any OCT signs of macular oedema varied depending on the criterion applied to define the eye as MI. The proportion was lowest for presence of haemorrhages at 1 disc diameter from the centre of the fovea at $23.2 \%$ (95\% CI, $16.6 \%$ $29.9 \%$ ) followed by $51.7 \%$ (95\% CI, 39.1\%-64.3\%) for the presence of exudates at the fovea, and 61.3\% (95\% CI, 53.1\%-69.5\%) for the presence of simultaneous haemorrhages and exudates (Chi squared $=45.3$, $\mathrm{P}<0.001)$.

Of the 47 eyes with a CMT of $\geq 300 \mu \mathrm{m}, 95.7 \%$ were noted to have at least one OCT sign of DME, which was a significantly higher proportion than in eyes with CMT of $<300 \mu \mathrm{m}(34.8 \%, \mathrm{P}<0.001$; Table 2).

The PPV of the DME screening was $13.4 \%$ (95\% CI, 9.8\%-17.0\%) and false-positive rate was $86.6 \%$ (95\% CI, 83.0\%-90.2\%) if macular thickness was used to define the presence of macular oedema. The PPV remained as low as $42.9 \%$ (95\% CI, 37.7\%-48.1\%) and false-positive rate $57.1 \%$ (95\% CI, 51.9\%-62.3\%) even if the thickness criterion was dropped and presence of OCT signs of macular oedema were considered sufficient to indicate the presence of oedema.

\section{Discussion}

Annual DR screening by ophthalmologists is an ideal but costly method that most health care systems can ill afford. The UK and Hong Kong adopt the fundus photography screening strategy that effectively prevents vision loss from PDR but may not be as accurate as in the screening of DME. The current study showed a high false-positive rate of $86.6 \%$ and low PPV of $13.4 \%$ in the screening for DME. Similar to our findings, a UK audit by Jyothi et $\mathrm{al}^{24}$ revealed that $79 \%$ of their M1 patients who were referred to specialist clinics did not require any intervention. Because a grade of $M 1$ is used to estimate the presence of CSME and, ideally, all CSME patients should be treated, most of those who were not treated would be due to a false positive result (ie patients without CSME being graded as M1). Therefore, despite the absence of further evaluation of their M1 patients, the results of Jyothi et al's study ${ }^{24}$ imply a low accuracy of the screening strategy.

To date, there is no consensus on the upper limit of normality for OCT central subfield (area within $500 \mu \mathrm{m}$ from the centre of the fovea) thickness, but it is thought to range from $230-300 \mu \mathrm{m}$ for time-domain OCT and 300-350 $\mu \mathrm{m}$ for sd-OCT. ${ }^{26}$ The difference between the two types of OCT machines arises because time-domain machines measure retinal thickness from the ellipsoid zone to the ILM while the spectral-domain machines use the distance between retinal pigment epithelium or Bruch's membrane to the ILM, which are more posterior structures to the ellipsoid zone. Most benchmark studies of the effects of intravitreal anti-VEGF injections in the management of DME used time-domain OCT for assessment. The upper limit of normal CMT was defined as $250 \mu \mathrm{m}$ in the Diabetic Retinopathy Clinical Research Network (DRCR Network) study ${ }^{10}$ and READ-2 study ${ }^{8} ; 275 \mu \mathrm{m}$ in the RISE and RIDE studies $^{7}$ and the RESTORE study ${ }^{9}$; and $300 \mu \mathrm{m}$ in the RESOLVE study. ${ }^{14}$ The DRCR Network also showed that sd-OCT measurement can be reliably converted to standard Stratus time-domain OCT measurement with conversion equations. ${ }^{13}$ If CMT of $250 \mu \mathrm{m}$ in time-domain OCT is converted to the sd-OCT, it will range from $290.2 \mu \mathrm{m}$ to $313.4 \mu \mathrm{m}$. We chose $300 \mu \mathrm{m}$ as the cut-off value for the upper limit of normal macular thickness to distinguish abnormal from normal because our Carl Zeiss Cirrus OCT is a sd-OCT. Similar cut-off values were adopted by the DRCR Network in a recently published paper. ${ }^{12}$ In their multicentre study, when Cirrus OCT was used, $305 \mu \mathrm{m}$ and $290 \mu \mathrm{m}$ were used to define increased CMT for males and females, respectively. ${ }^{12}$ Using $300 \mu \mathrm{m}$ as the cut-off in our reference standard gave a smaller number of false-positive diagnoses by traditional fundus photography screening than using a higher cut-off value, therefore favouring the current screening programme by being conservative in the estimation of false-positive rate. Another reason for using this criterion was because of

TABLE 2. Comparison of incidence of OCT signs between CMT of $\geq 300 \mu \mathrm{m}$ and CMT of $<300 \mu \mathrm{m}$

\begin{tabular}{|c|c|c|c|c|c|c|c|}
\hline \multirow[t]{2}{*}{ M1 cases } & \multicolumn{6}{|c|}{ No. (\%) } & \multirow{2}{*}{$\begin{array}{c}\text { Mean }( \pm \text { SD) } \\
\text { CMT }(\mu \mathrm{m})\end{array}$} \\
\hline & Eyes & Cyst & Subretinal fluid & $\begin{array}{l}\text { Diffuse retinal } \\
\text { thickening }\end{array}$ & $\begin{array}{l}\text { ILM profile } \\
\text { change }\end{array}$ & $\begin{array}{l}\text { Any OCT } \\
\text { signs }\end{array}$ & \\
\hline $\mathrm{CMT} \geq 300 \mu \mathrm{m}$ & $47(13.4)$ & $38(80.9)$ & $3(6.4)$ & 24 (51.1) & $43(91.5)$ & 45 (95.7) & $369.21 \pm 79.24$ \\
\hline CMT $<300 \mu \mathrm{m}$ & $305(86.6)$ & $80(26.2)$ & $1(0.3)$ & $51(16.7)$ & $46(15.1)$ & 106 (34.8) & $249.08 \pm 25.74$ \\
\hline All & $352(100.0)$ & $118(33.5)$ & $4(1.1)$ & 75 (21.3) & 89 (25.3) & 151 (42.9) & $265.12 \pm 55.42$ \\
\hline
\end{tabular}

Abbreviations: CMT = central macular thickness; ILM = internal limiting membrane; $\mathrm{MI}=$ maculopathy; OCT = optical coherence tomography; SD = standard deviation 
the importance of the screening programme to be sufficiently sensitive to identify subtle disease states. Macular oedema is less likely to be present when CMT is $<300 \mu \mathrm{m}$. Macular oedema should be diagnosed only when a subject's CMT is $\geq 300 \mu \mathrm{m}$ and additional criteria are met. These criteria are as follows: the presence of intraretinal cysts, subretinal fluid and/or diffuse retinal oedema (retinal thickening with areas of reduced retinal reflectivity on OCT scans) on more than one scan, or any of the above associated with a change in the ILM contour (Fig 2), including increased CMT or loss of foveal contour. ${ }^{27} \mathrm{~A}$ qualitative and quantitative assessment of the macula with OCT can objectively diagnose or exclude macular oedema.

It is worth noting that some believe macular thickness should not be included as an OCT criterion for determining the presence of DME. These ophthalmologists think that as long as any OCT sign of DME (ie presence of intraretinal cyst, subretinal fluid, diffuse retinal thickening and/ or change in foveal contour) is present, thickening ensues regardless of CMT. Although we agree that OCT signs signify the presence of genuine oedema, we believe it is still essential to include CMT in the diagnostic criteria because the basis for ophthalmologists treating patients with DME came from the large-scale study performed by the ETDRS group. ${ }^{6}$ The ETDRS group has proven that only patients with CSME identified ophthalmoscopically by ophthalmologists will benefit from laser treatment compared with controls. Biomicroscopic assessment of DME by an ophthalmologist, however, is less sensitive than an OCT scan in diagnosing macular oedema when retinal thickening is mild. ${ }^{28,29}$ Therefore, for diabetic patients with a CMT of $<300 \mu \mathrm{m}$, evidence may not support treatment even if intraretinal cysts or other OCT signs of macular oedema are present, especially since laser and antiVEGF therapies have potential side-effects. As all of the latest studies to evaluate the effects of antiVEGF injections in the management of CSME included the CMT criteria when recruiting patients, it was appropriate to include the macular thickness criterion when setting our reference standard. In fact, Bandello et al $^{30}$ have performed a subgroup analysis with RESTORE study data and showed that treatment efficacy varied among patients with different CMT, in which the visual acuity gain after treatment was less in patients with baseline CMT of $\leq 300 \mu \mathrm{m}$ (time-domain OCT measurement) than for those with CMT of $>300 \mu \mathrm{m}$. Moreover, patients with better baseline visual acuity were more likely to experience visual acuity loss following laser monotherapy. This further justifies the need for the thickness criterion to be included when considering treatment.

If $\mathrm{CMT} \geq 300 \mu \mathrm{m}$ is considered genuine
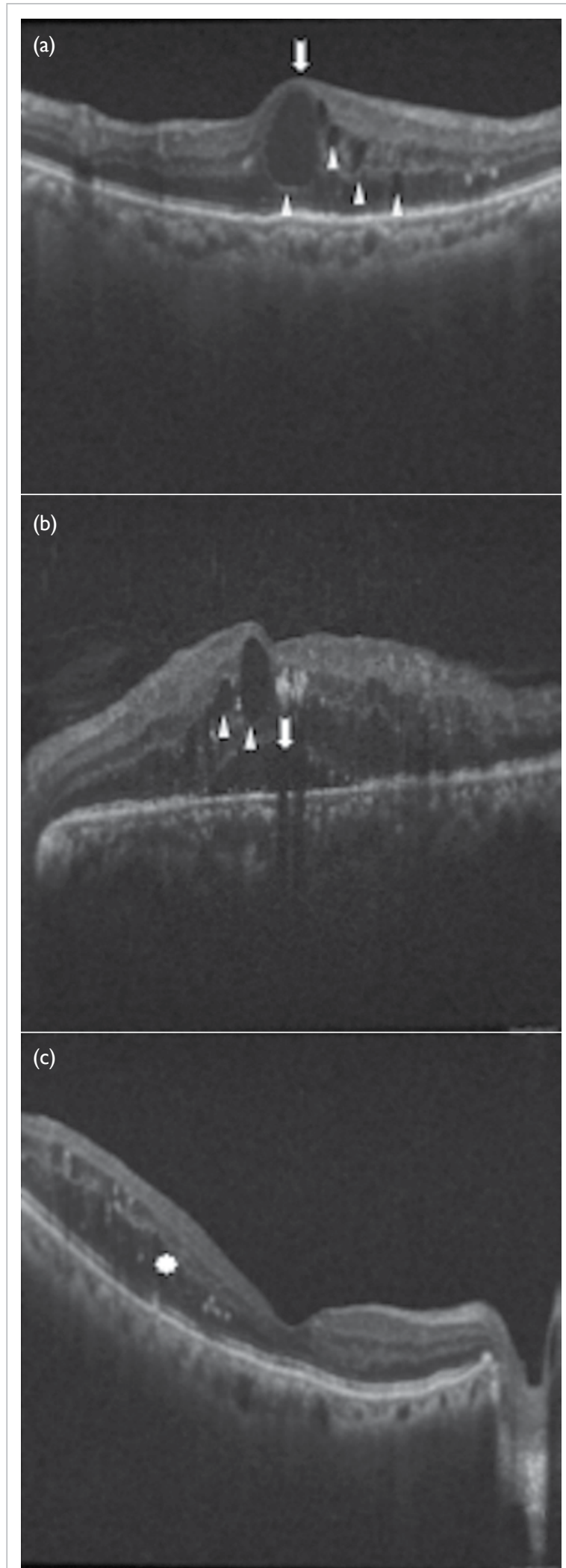

FIG 2. Optical coherence tomography scans of a patient with diabetic macular oedema: (a) presence of intraretinal cysts (arrowheads) and change in foveal ILM contour (arrow); (b) presence of subretinal fluid (arrow) and intraretinal cysts (arrowheads); and (c) presence of diffuse retinal thickening (asterisk)

Abbreviation: ILM = internal limiting membrane 
thickening of the macula, regardless of the presence of other OCT signs of DME, the false-positive rate of the current screening (proportion of referred M1 patients with CMT of $<300 \mu \mathrm{m}$ on OCT) protocol is $86.6 \%$. For every 1000 patients referred following screening to an ophthalmologist for diabetic maculopathy, 134 or fewer may require treatment because even among patients with increased CMT, the condition might not be clinically significant when it is only marginally greater than $300 \mu \mathrm{m}$. The cost of seeing one patient in a government eye clinic in Hong Kong is $\mathrm{HK} \$ 600$, and the marginal cost of offering one OCT scan is HK\$50 (cost of operating staff and colour print-out included; administrative costs in the health care system not included). Therefore, for every 1000 R1M1 patients offered OCT, at least 866 patients will have no CSME, thus referral to an eye specialist is unnecessary. In approximate monetary terms, hospitals would save HK\$469600 per 1000 R1M1 patients (866 x \$600 $1000 \times \$ 50)$ if they had an OCT machine. In addition to the financial burden, the high false-positive rate of screening would lead to unnecessary psychological stress for patients.

Based on our study data, if only OCT signs, not CMT, are taken as the reference standard for the presence of genuine DME, the false-positive rate of the current DME screening is also not low at 57.1\% of the screened-positive population.

A high false-positive rate of screening programmes places a huge burden on the health care system in terms of cost and manpower. In contrast, a high false-negative rate puts patients at risk of vision loss even when effective treatment is readily available. $^{31-33}$ An increased number of patients with vision loss as a consequence of false-negative screening will, in turn, translate into a financial burden on the health care system and society. In view of the rising prevalence of diabetes and its complications worldwide, ${ }^{34}$ a more reliable and costeffective screening strategy is needed.

We have reviewed the fundus photographs and OCT scans of R1M1 patients and endeavoured to determine why the PPV is unacceptably low. A substantial proportion of the false positive cases were graded M1 because of the presence of dot haemorrhages or microaneurysms within 1 disc diameter from the centre of the fovea together with a best corrected visual acuity of 6/12 or worse. This is one of the criteria for M1 grading in the protocol adopted by the Hong Kong RAMP-DR screening and the UK NHS Diabetic Eye Screening Programme. The inclusion of dot haemorrhages/ microaneurysms in the definition of M1 may not be beneficial to the screening programme. For example, they are not included in the Scottish Diabetic Retinopathy Screening Programme (Scottish Diabetic Retinopathy Grading Scheme 2007 v1.1).
Further studies should be conducted to evaluate the effects of amending the grading protocol of M1 (eg by revising the grading criteria) in the current screening strategy. The false-positive rate of screening may be reduced, perhaps with minimal impact on the false-negative rate. If resources are available, the addition of OCT imaging in selected cases (eg OCT scans for all patients graded as M1), or even for all (ie OCT for all in addition to fundus photography) may also help increase the effectiveness of screening. Either way, although the false-negative rate of DR screening might be increased, the consequence is not as severe in DME screening as other screenings because CSME generally impairs vision slowly. Furthermore, all negatively screened patients will be screened again in 1 year. If there is progression of disease, signs of disease, such as presence of exudate, will likely become more prominent and be noticed at the subsequent annual screenings. Subtle changes that cannot be detected by screening will not hugely affect the patient's vision. If the screening strategy is enhanced by performing additional OCT scans, there will be additional benefits on top of the improved accuracy in DME screening since OCT evidence of micro-structural changes to the retinal layers has been shown to correlate well with visual acuity and may have prognostic value in DME. ${ }^{35}$

Since this study recruited consecutive eligible patients from the diabetes complication screening programme and this screening programme is catered to all the public diabetic patients in the Hong Kong West Cluster, which is a representative population of Hong Kong, our findings should reflect the accuracy of the Hong Kong RAMP-DR screening programme.

Our study had several limitations, including potential selection bias due to subject recruitment solely in a public hospital, and self-selection bias due to refusal of eligible diabetic patients to participate in screening and/or screened-positive patients to participate in this study. There are a lack of accurate local epidemiological data regarding the prevalence of diabetes in the population resident in the catchment area of the screening programme and the proportion of all diabetic patients in the Hong Kong West Cluster (coverage area) who attend public services is unknown. Hence, our study subjects might not be representative of all diabetic patients in the study area. Nonetheless unlike voluntary response bias, when stratified to different severity levels (eg M0 or M1; R0, R1, R2, or R3), the presentation of DR differs little between patients in the public sector and private sector so bias should be minimal. Regarding self-selection bias, we have no data for the proportion of eligible patients who refused to participate in the screening programme. All patients who visited our clinic were those who agreed to the screening and had been referred from a general out-patient clinic or Department of Medicine 
of Queen Mary Hospital. All diabetic patients who are currently followed up in the public sector of Hong Kong West Cluster will attend the universal DR screening programme (RAMP). Since this is part of their diabetes follow-up, we may assume that only those who refuse such follow-up in the public sector will miss the RAMP screening. Therefore these potential sources of bias will not affect interpretation of our data. We have not documented the number of screened-positive subjects (DR grade R1M1) who refused to participate in our study, but we believe the number would have been small given our convenient location and the non-invasive nature of OCT scans, thus we should only expect minimal self-selection bias.

Another limitation of our study is that only one experienced retina specialist was responsible for determining the presence of OCT signs of DME in our subjects. Nonetheless the retina specialist was blinded to the fundus photography DR grading, and the presence of OCT signs such as intraretinal fluid and change in foveal contour were distinct and not ambiguous. As such, the lack of multiple independent investigators to determine the presence of OCT signs of macular oedema should not have induced bias or affected our findings and final analysis. This study also lacks the data regarding the false-negative rate in the current screening programme. Since the objective of our study was to evaluate the rate of false-positive referrals, only patients with eyes graded as M1 were recruited. In order to evaluate the screening system as a whole, analysis of the data of eyes graded as M0 is also essential. Moreover, the strength and weakness of the screening can be objectively assessed with the calculated sensitivity, specificity, positive and negative predictive values, and false-positive and false-negative rates. Further studies in this respect are warranted.

In our study, we used the Macular Cube protocol to measure CMT, determining the macular thickness at 128 different points in the foveal region $(500 \mu \mathrm{m}$ radius from the centre of fovea). By averaging the 128 readings, the CMT of one patient was obtained. This way of measuring CMT is more reliable than performing only two scans (horizontal and vertical) when evaluating macular oedema with OCT.

\section{Conclusion}

The low PPV of the current DME screening adopted by the UK and Hong Kong will lead to unnecessary psychological stress for patients and place a financial burden on the health care system. An improved screening protocol, such as the addition of sd-OCT scans in selected patients or amendment of the grading protocol of the current screening programme, is necessary to improve its costeffectiveness.

\section{Acknowledgements}

This study was supported by the Department of Ophthalmology, The University of Hong Kong. The authors would like to thank all the ophthalmologists and physicians at Queen Mary Hospital (Hong Kong) who were involved in management of the patients.

\section{Declaration}

All authors have disclosed no conflicts of interest.

\section{References}

1. Diabetic retinopathy. Preferred practice pattern guidelines. Available from: https://www.aao.org/about-preferredpractice-patterns. Accessed 28 Nov 2016.

2. Klein R, Klein BE, Moss SE, Davis MD, DeMets DL. The Wisconsin epidemiologic study of diabetic retinopathy. III. Prevalence and risk of diabetic retinopathy when age at diagnosis is 30 or more years. Arch Ophthalmol 1984;102:527-32.

3. Klein R, Klein BE, Moss SE, Davis MD, DeMets DL. The Wisconsin epidemiologic study of diabetic retinopathy. II. Prevalence and risk of diabetic retinopathy when age at diagnosis is less than 30 years. Arch Ophthalmol 1984;102:520-6.

4. Aiello LM. Perspectives on diabetic retinopathy. Am J Ophthalmol 2003;136:122-35.

5. Aiello LP, Gardner TW, King GL, et al. Diabetic retinopathy. Diabetes Care 1998;21:143-56.

6. Photocoagulation for diabetic macular oedema. Early Treatment Diabetic Retinopathy Study report number 1. Early Treatment Diabetic Retinopathy Study research group. Arch Ophthalmol 1985;103:1796-806.

7. Nguyen QD, Brown DM, Marcus DM, et al. Ranibizumab for diabetic macular edema: results from 2 phase III randomized trials: RISE and RIDE. Ophthalmology 2012;119:789-801.

8. Nguyen QD, Shah SM, Khwaja AA, et al. Two-year outcomes of the ranibizumab for edema of the macula in diabetes (READ-2) study. Ophthalmology 2010;117:214651.

9. Lang GE, Berta A, Eldem BM, et al. Two-year safety and efficacy of ranibizumab $0.5 \mathrm{mg}$ in diabetic macular edema: interim analysis of the RESTORE extension study. Ophthalmology 2013;120:2004-12.

10. Elman MJ, Bressler NM, Qin H, et al. Expanded 2-year follow-up of ranibizumab plus prompt or deferred laser or triamcinolone plus prompt laser for diabetic macular edema. Ophthalmology 2011;118:609-14.

11. Cheung N, Wong IY, Wong TY. Ocular anti-VEGF therapy for diabetic retinopathy: overview of clinical efficacy and evolving applications. Diabetes Care 2014;37:900-5.

12. Diabetic Retinopathy Clinical Research Network, Wells JA, Glassman AR, et al. Aflibercept, bevacizumab, or ranibizumab for diabetic macular edema. N Engl J Med 2015;372:1193-203.

13. Diabetic Retinopathy Clinical Research Network Writing Committee, Bressler SB, Edwards AR, et al. Reproducibility of spectral-domain optical coherence tomography retinal thickness measurements and conversion to equivalent time-domain metrics in diabetic macular edema. JAMA Ophthalmol 2014;132:1113-22. 
14. Massin P, Bandello F, Garweg JG, et al. Safety and efficacy of ranibizumab in diabetic macular edema (RESOLVE Study): a 12-month, randomized, controlled, double-masked, multicenter phase II study. Diabetes Care 2010;33:2399405.

15. Scotland GS, McNamee P, Philip S, et al. Cost-effectiveness of implementing automated grading within the national screening programme for diabetic retinopathy in Scotland. Br J Ophthalmol 2007;91:1518-23.

16. Kawasaki R, Akune Y, Hiratsuka Y, Fukuhara S, Yamada M. Cost-utility analysis of screening for diabetic retinopathy in Japan: a probabilistic Markov modeling study. Ophthalmic Epidemiol 2015;22:4-12.

17. Rachapelle S, Legood R, Alavi Y, et al. The cost-utility of telemedicine to screen for diabetic retinopathy in India. Ophthalmology 2013;120:566-73.

18. Tung TH, Shih HC, Chen SJ, Chou P, Liu CM, Liu JH. Economic evaluation of screening for diabetic retinopathy among Chinese type 2 diabetics: a community-based study in Kinmen, Taiwan. J Epidemiol 2008;18:225-33.

19. Stefánsson E, Bek T, Porta M, Larsen N, Kristinsson JK, Agardh E. Screening and prevention of diabetic blindness. Acta Ophthalmol Scand 2000;78:374-85.

20. Lin DY, Blumenkranz MS, Brothers RJ, Grosvenor DM. The sensitivity and specificity of single-field nonmydriatic monochromatic digital fundus photography with remote image interpretation for diabetic retinopathy screening: a comparison with ophthalmoscopy and standardized mydriatic color photography. Am J Ophthalmol 2002;134:204-13.

21. James M, Turner DA, Broadbent DM, Vora J, Harding SP. Cost effectiveness analysis of screening for sight threatening diabetic eye disease. BMJ 2000;320:1627-31.

22. Prescott G, Sharp P, Goatman K, et al. Improving the cost-effectiveness of photographic screening for diabetic macular oedema: a prospective, multi-centre, UK study. Br J Ophthalmol 2014;98:1042-9.

23. Bresnick GH, Mukamel DB, Dickinson JC, Cole DR. A screening approach to the surveillance of patients with diabetes for the presence of vision-threatening retinopathy. Ophthalmology 2000;107:19-24.

24. Jyothi S, Elahi B, Srivastava A, Poole M, Nagi D, Sivaprasad $\mathrm{S}$. Compliance with the quality standards of National Diabetic Retinopathy Screening Committee. Prim Care
Diabetes 2009;3:67-72.

25. Lian JX, Gangwani RA, McGhee SM, et al. Systematic screening for diabetic retinopathy (DR) in Hong Kong: prevalence of DR and visual impairment among diabetic population. Br J Ophthalmol 2016;100:151-5.

26. Virgili G, Menchini F, Casazza G, et al. Optical coherence tomography (OCT) for detection of macular oedema in patients with diabetic retinopathy. Cochrane Database Syst Rev 2015;(1):CD008081.

27. Mackenzie S, Schmermer C, Charnley A, et al. SDOCT imaging to identify macular pathology in patients diagnosed with diabetic maculopathy by a digital photographic retinal screening programme. PloS One 2011;6:e14811.

28. Brown JC, Solomon SD, Bressler SB, Schachat AP, DiBernardo C, Bressler NM. Detection of diabetic foveal edema: contact lens biomicroscopy compared with optical coherence tomography. Arch Ophthalmol 2004;122:330-5.

29. Browning DJ, McOwen MD, Bowen RM Jr, O'Marah TL. Comparison of the clinical diagnosis of diabetic macular edema with diagnosis by optical coherence tomography. Ophthalmology 2004;111:712-5.

30. Bandello F, Cunha-Vaz J, Chong NV, et al. New approaches for the treatment of diabetic macular oedema: recommendations by an expert panel. Eye (Lond) 2012;26:485-93.

31. Focal photocoagulation treatment of diabetic macular edema. Relationship of treatment effect to fluorescein angiographic and other retinal characteristics at baseline: ETDRS report no. 19. Early Treatment Diabetic Retinopathy Study Research Group. Arch Ophthalmol 1995;113:1144-55.

32. Goyal S, Lavalley M, Subramanian ML. Meta-analysis and review on the effect of bevacizumab in diabetic macular edema. Graefes Arch Clin Exp Ophthalmol 2011;249:1527.

33. Abu El-Asrar AM, Al-Mezaine HS. Advances in the treatment of diabetic retinopathy. Discov Med 2010;9:36373.

34. Wild S, Roglic G, Green A, Sicree R, King H. Global prevalence of diabetes: estimates for the year 2000 and projections for 2030. Diabetes Care 2004;27:1047-53.

35. Wong RL, Lee JW, Yau GS, Wong IY. Relationship between outer retinal layers thickness and visual acuity in diabetic macular edema. Biomed Res Int 2015;2015:981471. 